\title{
Predicting neuronal activity with simple models of the threshold type: Adaptive Exponential Integrate-and-Fire model with two compartments
}

\author{
Claudia Clopath ${ }^{\mathrm{a}, *}$, Renaud Jolivet ${ }^{\mathrm{a}}$, Alexander Rauch ${ }^{\mathrm{b}, \mathrm{c}}$, \\ Hans-Rudolf Lüscher ${ }^{\mathrm{c}}$, Wulfram Gerstner ${ }^{\mathrm{a}}$ \\ ${ }^{a}$ School of Computer and Communication Sciences and Brain Mind Institute, EPFL, CH-1015 Lausanne, Switzerland \\ ${ }^{\mathrm{b}}$ Max-Plank-Institute for Biological Cybernetics, D-72012 Tübingen, Germany \\ ${ }^{\mathrm{c}}$ Institute of Physiology, University of Bern, CH-3012 Bern, Switzerland
}

\begin{abstract}
An adaptive Exponential Integrate-and-Fire (aEIF) model was used to predict the activity of layer-V-pyramidal neurons of rat neocortex under random current injection. A new protocol has been developed to extract the parameters of the aEIF model using an optimal filtering technique combined with a black-box numerical optimization. We found that the aEIF model is able to accurately predict both subthreshold fluctuations and the exact timing of spikes, reasonably close to the limits imposed by the intrinsic reliability of pyramidal neurons.
\end{abstract}

(C) 2006 Elsevier B.V. All rights reserved.

PACS: 87.19.La

Keywords: Adaptation; Exponential integrate-and-fire; Neuron; Spike timing

\section{Introduction}

Electrophysiological data can be described by detailed conductance-based models (Hodgkin-Huxley-type models [6]). However, those models are rather complex, which implies that they are difficult to analyze and costly to implement numerically. Moreover, it is unclear how many details of conductance-based models are really necessary for the reproduction of experimental spike patterns $[4,13]$. For those reasons, simple phenomenological spiking neurons such as Integrate-and-Fire models are highly popular.

The adaptive Exponential Integrate-and-Fire (aEIF) model used in this paper generalizes the standard leaky Integrate-and-Fire model in several directions: the strict threshold is replaced by a more realistic smooth threshold zone as in the Exponential Integrate-and-Fire neuron [2]. Furthermore, addition of a second variable captures subthreshold resonance or adaptation [7,15]. The aEIF

\footnotetext{
*Corresponding author.

E-mail address: claudia.clopath@epfl.ch (C. Clopath).
}

model showed convincing performances when compared to more detailed models [1], but so far, has never been tested on recordings of real neuron.

In this report, we will test the performances of the aEIF model on layer- $\mathrm{V}$ neocortical pyramidal neurons under random current injection.

\section{Model}

The aEIF is defined by [1]

$$
\begin{aligned}
C \frac{\mathrm{d} u(t)}{\mathrm{d} t}= & -g_{\mathrm{L}}\left(u(t)-E_{\mathrm{L}}\right) \\
& +g_{\mathrm{L}} \Delta_{\mathrm{T}} \exp \left(\frac{u(t)-V_{\mathrm{T}}}{\Delta_{\mathrm{T}}}\right)-w+I, \\
\tau_{w} \frac{\mathrm{d} w(t)}{\mathrm{d} t}= & a\left(u(t)-E_{\mathrm{L}}\right)-w(t),
\end{aligned}
$$

where $C$ is the membrane capacitance, $g_{\mathrm{L}}$ the leak conductance, $E_{\mathrm{L}}$ the resting potential, $\Delta_{\mathrm{T}}$ the slope factor and $V_{\mathrm{T}}$ the threshold potential (Fig. 1). Note that formally, $E_{\mathrm{L}}$ is not exactly the resting potential because of the 


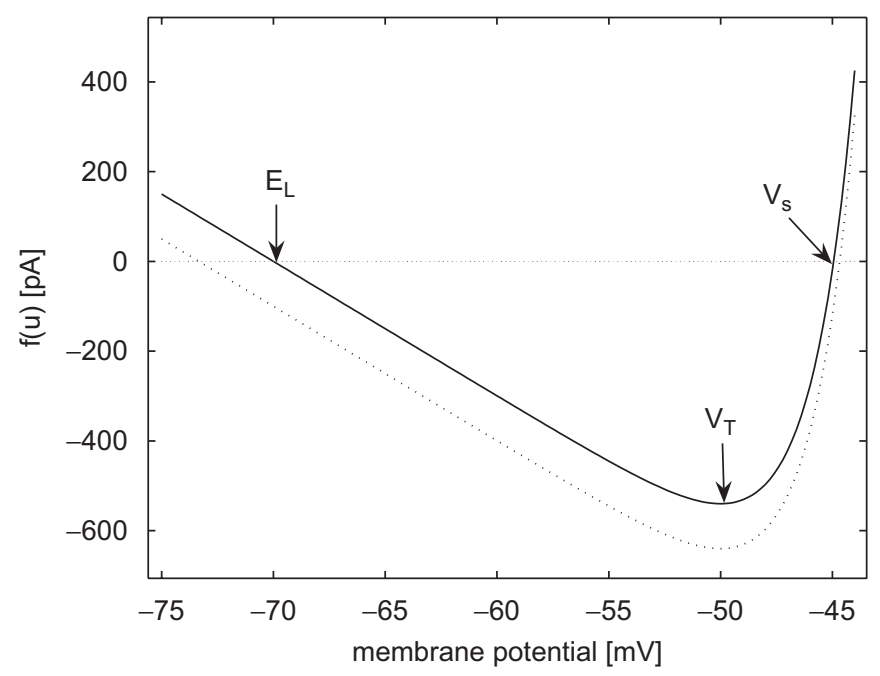

Fig. 1. Spike function $f(u)=C \mathrm{~d} u / \mathrm{d} t-I$ of the aEIF model in the nonadapted state ( $w=0$; black line). The left intersection of the spike function with the horizontal axis is the resting potential $E_{\mathrm{L}}$, the right intersection gives the potential $V_{\mathrm{s}}$ above which the spike is generated. The minimum of $f(u), V_{\mathrm{T}}$, gives the maximum subthreshold potential that can be reached by constant current injection. In the adapted state $(w>0)$, the spike function $f(u)=C \mathrm{~d} u / \mathrm{d} t-I$ is shifted vertically downward (dotted line).

exponential term. The variable $w$ describes the level of adaptation of the neuron and $a$ represents the relevance of subthreshold adaptation. The exponential term describes the early activation of voltage-gated sodium channels.

Formally the model is said to generate a spike if the potential $u$ grows rapidly to infinity. In practice, a spike event is recorded when the voltage reaches a threshold $V_{\text {peak }}=20 \mathrm{mV}$. The exact value is not critical because $V_{\text {peak }}$ only shifts spike times by a fraction of millisecond. After the spike has been triggered, $u$ is reset to the resting potential $E_{\mathrm{L}}$ and the variable $w$ is increased by an amount $b$, which accounts for spike-triggered adaptation.

The original aEIF model is a point neuron model i.e. without spatial structure. However, in this study, we decided to take into account the coupling of the soma with the dendrites. Therefore, we used a two-compartment model (one somatic compartment coupled to a passive dendritic compartment) defined by

$$
\begin{aligned}
& C \frac{\mathrm{d} u_{s}}{\mathrm{~d} t}=-g_{\mathrm{L}}\left(u_{\mathrm{s}}-E_{\mathrm{L}}\right)-\frac{g_{\mathrm{c}}}{p}\left(u_{\mathrm{s}}-u_{\mathrm{d}}\right) \\
&+g_{\mathrm{L}} \Delta_{\mathrm{T}} \exp \left(\frac{u_{\mathrm{s}}-V_{\mathrm{T}}}{\Delta_{\mathrm{T}}}\right)-w+I, \\
& C \frac{\mathrm{d} u_{\mathrm{d}}}{\mathrm{d} t}=-g_{\mathrm{L}}\left(u_{\mathrm{d}}-E_{\mathrm{L}}\right)-\frac{g_{\mathrm{c}}}{1-p}\left(u_{\mathrm{d}}-u_{\mathrm{s}}\right), \\
& \tau_{w} \frac{\mathrm{d} w}{\mathrm{~d} t}=a\left(u_{\mathrm{s}}-E_{\mathrm{L}}\right)-w,
\end{aligned}
$$

where $u_{\mathrm{s}}$ is the membrane voltage in the somatic compartment, $u_{\mathrm{d}}$ the membrane voltage in the dendritic compartment, $g_{\mathrm{c}}$ the coupling conductance and $p=$ somatic area/ total area. The two-compartment model is motivated by experimental results. Indeed, the linear response kernel is best fitted by a double exponential (see below point 3(i)), suggesting a coupling between soma and a passive dendrite acting as current sink [8].

\section{Parameter fitting}

Recordings of layer- $\mathrm{V}$ pyramidal neurons of rat neocortex were used to determine parameters of the model. The neurons were recorded intracellularly in vitro while stimulated at the soma by a randomly fluctuating current generated by an Ornstein-Uhlenbeck (OU) process (autocorrelation time $1 \mathrm{~ms}$ ). Both mean and variance of the $\mathrm{OU}$ process were varied in order to sample the response of the neurons to various levels of tonic and time-dependent inputs. Details of the experimental procedure can be found in [14].

Our method to extract the parameters of the aEIF model is based on the following steps:

(i) Passive membrane properties $\left(C, g_{\mathrm{L}}, g_{\mathrm{c}}, p, E_{\mathrm{L}}\right)$ : In subthreshold regime (where the exponential term can be neglected), Eqs. (3) and (4) can be integrated [3],

$$
u(t)=\int_{0}^{+\infty} \kappa_{\infty}(s) I(t-s) \mathrm{d} s
$$

In the non-adapted state $w=0$, we find

$\kappa_{\infty}(s)=\frac{1}{C}\left[p \mathrm{e}^{-s / \tau_{s}}+(1-p) \mathrm{e}^{-s / \tau_{c}}\right]$,

where $\tau_{\mathrm{s}}=C / g_{\mathrm{L}}$ is the somatic membrane time constant and $\tau_{\mathrm{c}}=[p(p-1) C] /\left[p(p-1) g_{\mathrm{L}}-g_{\mathrm{c}}\right]$ is the coupling time constant.The kernel $\kappa_{\infty}$ is extracted by the Wiener-Hopf optimal filtering technique [8]. This step involves a comparison of the subthreshold fluctuations with the corresponding input current. This yields a "raw" filter $\kappa_{\text {exp }}$ (Fig. 2). The filter $\kappa_{\text {exp }}$ is well fitted by the double exponential $\kappa_{\infty}$ derived from our two-compartment model. $C, g_{\mathrm{L}}, g_{\mathrm{c}}, p$ were extracted from the double exponential fit $\kappa_{\infty}$ (Eq. (7)) of $\kappa_{\text {exp }}, E_{\mathrm{L}}$ from the resting value at the beginning of the recording.

(ii) Slope factor: The slope factor determines the sharpness of the threshold. In the limit $\Delta_{\mathrm{T}} \rightarrow 0$, the model becomes a standard leaky Integrate-and-Fire model. As the threshold has a region of fuzziness, we decided to fix the slope factor at $\Delta_{\mathrm{T}}=2 \mathrm{mV}$ so as to restrict the number of parameters to be optimized. This value seems reasonable and is close to the value found for the Wang-Buszaki model [2].

(iii) Subthreshold adaptation: According to systems theory, it is not possible to extract the subthreshold adaptation $a$ from our data set. Therefore, we set $a$ to zero. Indeed, the Laplace transformed system has one pole and one zero that masque each other (i.e. the determinant of the identificability matrix is 


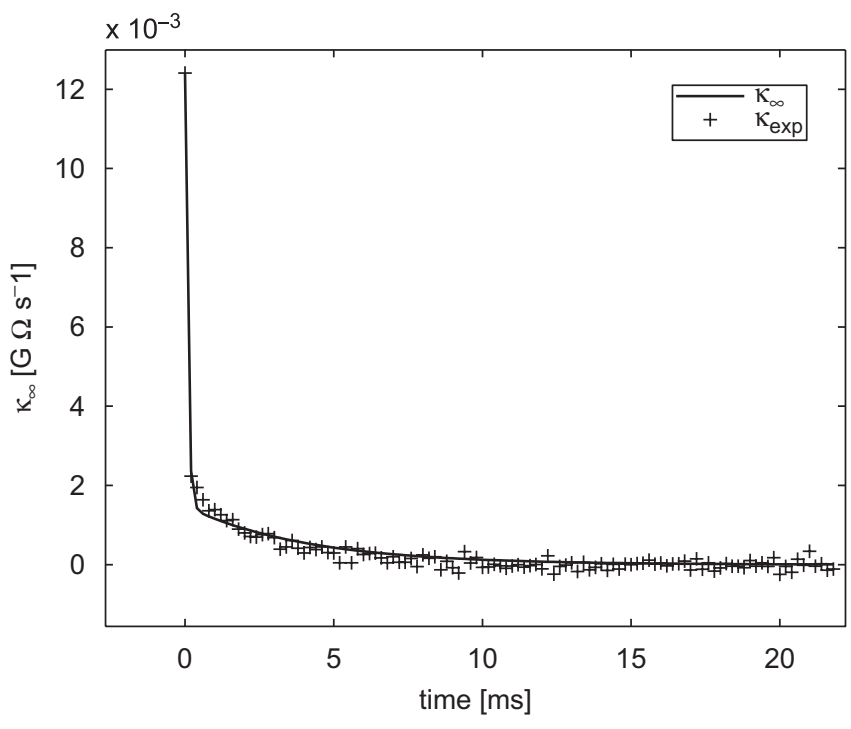

Fig. 2. Raw data of the kernel $\kappa_{\text {exp }}$ extracted by optimal filtering technique (symbols) have been fitted by the double exponential $\kappa_{\infty}$ (solid line).

close to zero), preventing the system to be fully characterizable [10].

(iv) Voltage reset: After a spike has been triggered, the voltage is simply reset to the resting potential $E_{\mathrm{L}}$.

(v) Optimization: Finally, the remaining parameters, $V_{\mathrm{T}}$, $\tau_{w}, b$ were optimized using the simulated annealing technique optimizing the firing rate and maximizing the coincidence factor $\Gamma_{n \rightarrow m}$. We minimized the following expression:

$2\left|\frac{v_{\text {data }}-v_{\text {sim }}}{v_{\text {data }}}\right|-\Gamma_{n \rightarrow m}$

where $v_{\text {data }}$ is the firing rate of the neuron data and $v_{\text {sim }}$ is the firing rate of the simulated data. $\Gamma_{n \rightarrow m}$ is defined by [9]

$\Gamma_{n \rightarrow m}=\frac{N_{\text {coinc }}-\left\langle N_{\text {coinc }}\right\rangle}{\frac{1}{2}\left(N_{\text {data }}+N_{\text {aEIF }}\right)} \frac{1}{N}$,

where $N_{\text {data }}$ is the number of spikes in the reference spike train (recordings of pyramidal cells), $N_{\mathrm{aEIF}}$ is the number of spikes in the predicted spike train (generated with the aEIF model with the same driving current). $N_{\text {coinc }}$ is the number of coincident spikes with precision $\Delta=2 \mathrm{~ms}$ and $\left\langle N_{\text {coinc }}\right\rangle$ is the number of coincidences generated by a homogeneous Poisson process with the same rate $v_{\text {sim }}$ as the spike train generated with the aEIF model. Finally, the normalization $N=1-2 v_{\mathrm{sim}} \Delta$ allows $\Gamma_{n \rightarrow m}$ to reach 1 only if the spike train of the aEIF model reproduces exactly the spike train of the cell. $\Gamma_{n \rightarrow m}$ will be 0 if the similarity between the two spike trains is not better than between that two random spike trains generated by homogeneous Poisson processes at the same rate. In order to test the robustness of the method, we picked one cell and repeated the parameter optimization by simulated annealing 10 times. We found that the $V_{\mathrm{T}}$ is very robust within errors less than $3 \%$. The parameters $b$ and $\tau_{w}$ are strongly correlated. While individual variance is high their product $b \tau_{w}$ is stable.

\section{Results}

The data set consists of four different neurons. For each cell, a set of 10 different input currents with different means and variances are injected. Each input trace is repeated four times. Fig. 3 shows a direct comparison between predicted and recorded spike trains for a typical neuron. Both spike trains are almost indistinguishable (Fig. 3A; for clarity reasons, the predicted spike train has been shifted upward). Even when zooming in the subthreshold regime, differences are in the range of a few millivolts only (Fig. 3B). The spike dynamics is correctly predicted apart from a short period of time just after the spike is emitted (Fig. 3C). This is due to the reset value of the voltage which is set to the resting potential.

The model performances were evaluated using the coincidence factor $\Gamma_{n \rightarrow m}$ (Eq. (8)). Our model is facing natural limits of prediction because cortical pyramidal neurons respond with very different reliability depending on the type of stimulation they receive [11]. As we cannot expect our model to yield better predictions than the intrinsic reliability of the neuron, we consider the intrinsic reliability of the neuron as an upper bound. The intrinsic reliability can be easily measured since the same input has been injected four times in the same cell. The reliability of neurons does not vary significantly with the mean of the injected current. However, it strongly depends on the variance of the current $[8,11]$. In the case of low variance, the spike timing is not controlled by the stimulus anymore. Therefore, we abandon the data with low variance $(\sigma<150 \mathrm{pA})$. Intrinsic reliability is characterized by the factor $\Gamma_{n \rightarrow n}$ in analogy to Eq. (8). The subscript $n \rightarrow n$ means that the neuron is compared to itself across two different trials with the same realization of the input. We remark that data with high variance input are more likely to resemble an in vivo situation than low variance input data. For data used below, the intrinsic reliability varies from $\Gamma_{n \rightarrow n}=75 \%$ to as low as $\Gamma_{n \rightarrow n}=20 \%$.

We found that the aEIF model predicts up to $\Gamma_{\text {eff }}=$ $96 \%$ of the spikes that can be predicted $\left(\Gamma_{\text {eff }}=\right.$ $\Gamma_{n \rightarrow m} / \Gamma_{n \rightarrow n}, m \rightarrow n$ means model compared to neuron) and on average $\Gamma_{\text {eff }}=60 \%$ (Fig. 4).

Fig. 5 shows the experimental spike trains during four repetitions with the same driving current (bottom traces) as well as the simulated spike train (top trace) for different reliability and performance cases.

\section{Discussion and conclusion}

We tested the aEIF model on experimental electrophysiology recordings and found a prediction of the spike 


\section{A}

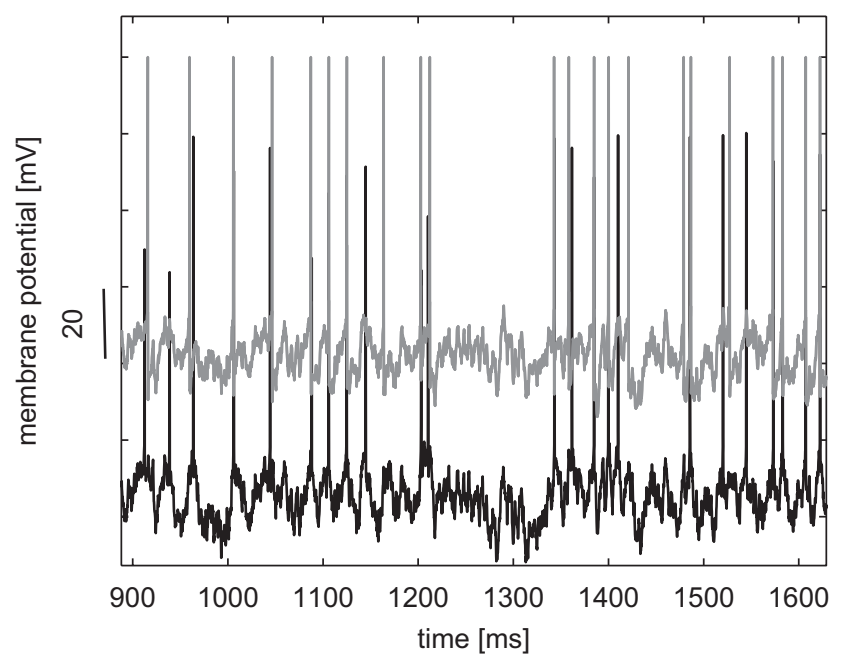

B

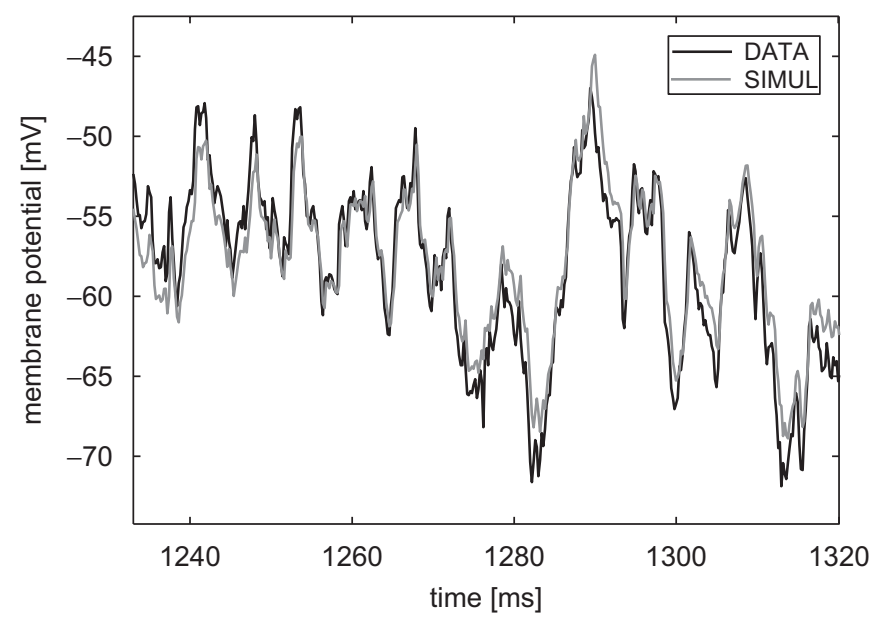

C

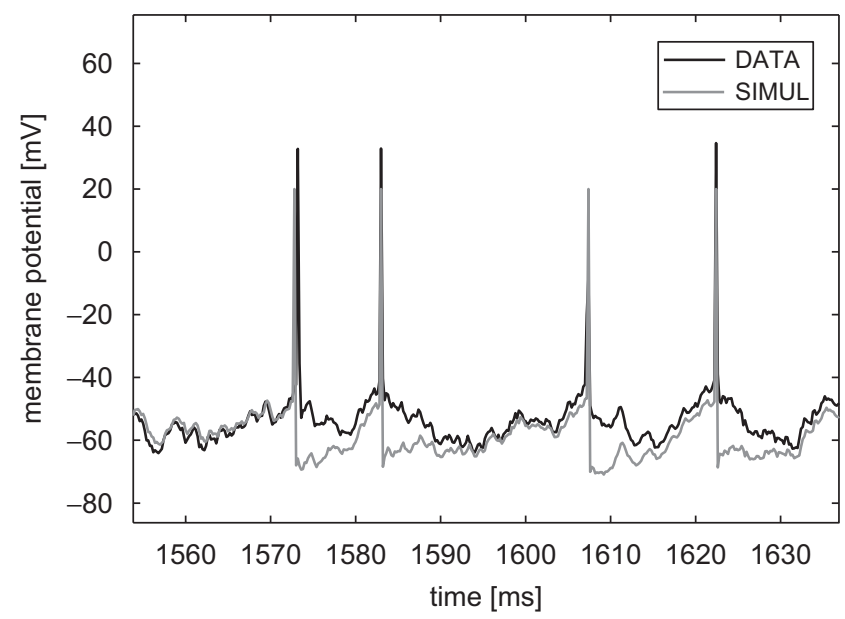

Fig. 3. Performances of the aEIF model. A. Predictions of the model (grey line) are compared to the spike train of the corresponding neuron (black line). For this graph only, the simulated trace has been shifted by $40 \mathrm{mV}$ upward for reasons of visual clarity. B. Zoom on the subthreshold regime. C. Zoom on four correctly predicted spikes.

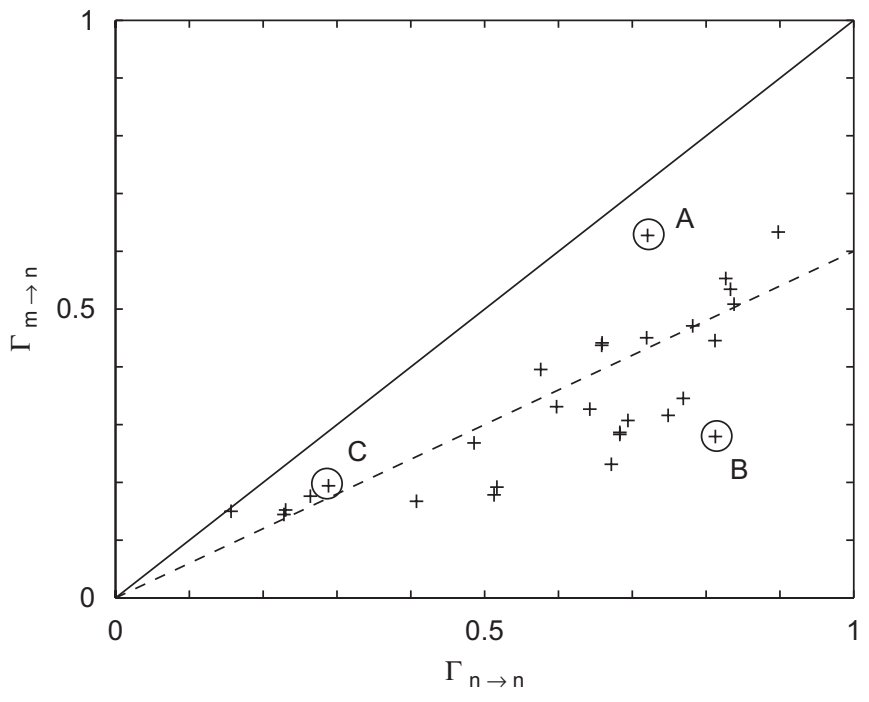

Fig. 4. The performance of the aEIF $\left(\Gamma_{m \rightarrow n}\right)$ is plotted versus the intrinsic reliability $\left(\Gamma_{n \rightarrow n}\right)$ for each data set. The diagonal yields the upper bound of the model. The average prediction is shown by the dashed line ( $\Gamma_{\text {eff }}=\Gamma_{n \rightarrow m} / \Gamma_{n \rightarrow n}=0.6$ ). More details on the specific cases $A, B, C$ are shown in Fig. 5.

times $\Gamma_{\text {eff }}$ up to $96 \%$ (average of $60 \%$ ). With the same data set, a Spike Response Model with dynamic threshold has been evaluated and the performances were $\Gamma_{\text {eff }}$ up to $75 \%$ (average 65\%) [8].

We remark that the protocol used for the recordings is not the most suitable for characterizing our model: in our extraction method, we had to set the subthreshold adaptation $a$ to 0 . In addition, data generated purely by simulation of the aEIF model were characterized very badly with our method (average of $\Gamma=85 \%$ ). A completely different protocol to extract the parameters of the aEIF model has been proposed recently by Brette and Gestner [1]. This protocol contains a series of standard electrophysiological paradigms (injection of current pulses, slow current ramps and random conductance injections). It has been tested with data generated by a detailed model and yielded excellent performances $(\Gamma=96 \%)$. In addition, this protocol allows to reduce noise (averaging over several recordings), so that the subthreshold adaption $a$ could, in principal, be extracted from pyramidal cell recordings. The latter protocol is under study at the moment using a new data set recorded following the methodology proposed by Brette and Gerstner [1].

In the aEIF model, adaptation is a useful component since it allows the model to account for different driving regimes. We found as well that it is relatively easy to correctly predict the subthreshold dynamics even with a simple leaky integrator but it is difficult to find an efficient threshold criterion for spike initiation. This problem is solved by the aEIF model which includes an additional exponential term to describe early activation of voltagegated sodium channels. This last addition allows to model 
specific behaviors like delayed spike initiation and offers flexibility at the level of the threshold mechanism. It was recently suggested by Naundorf and colleagues that the

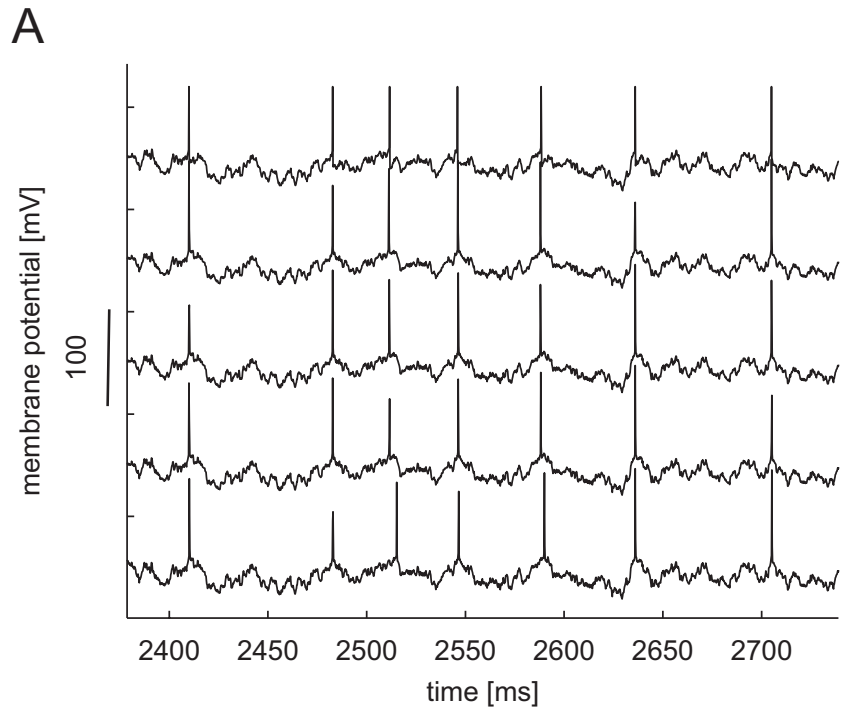

B

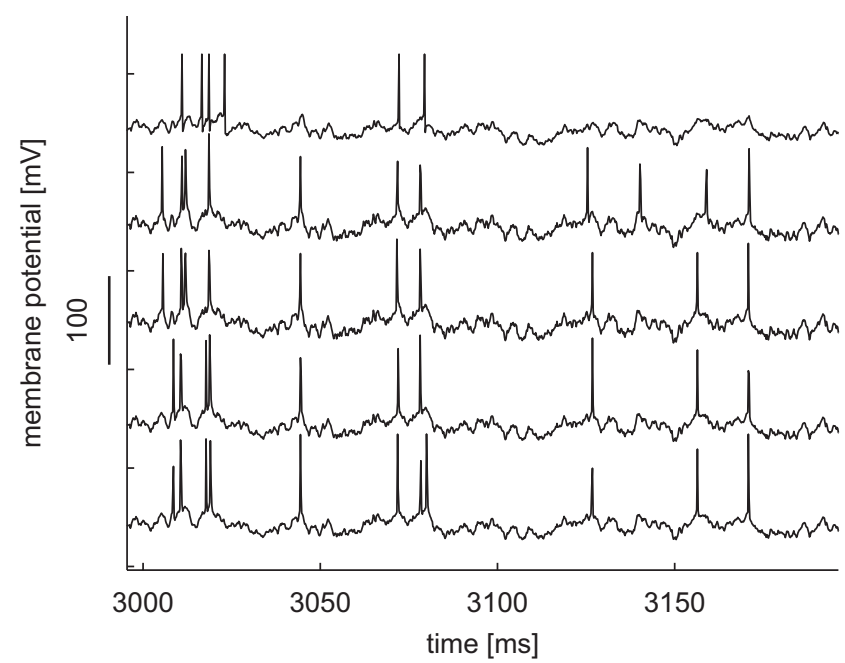

C

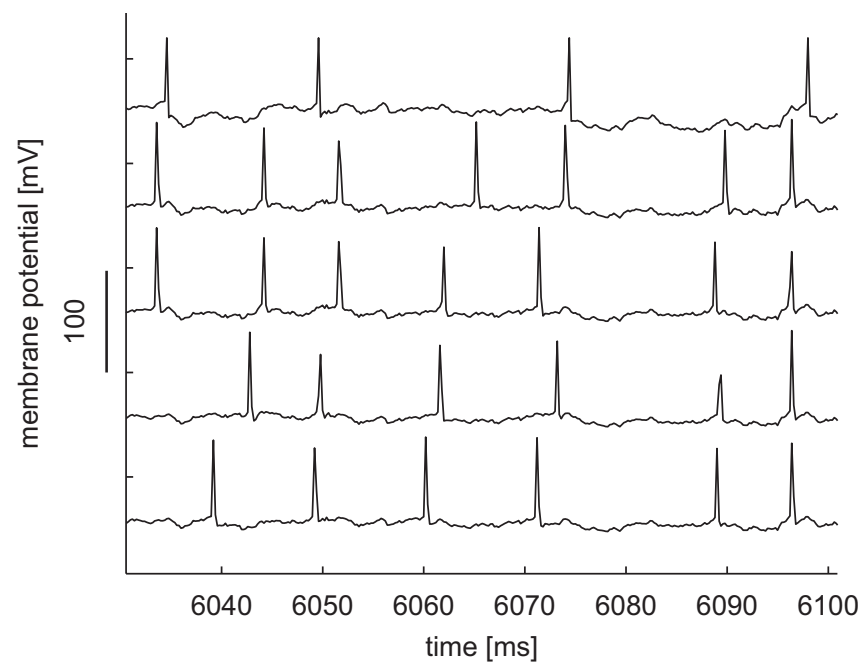

rapid dynamics of action potential initiation in cortical neurons are outside the range of behaviors described by the classical Hodgkin-Huxley theory [12]. In the aEIF model, the exponential term allows a fast activation of the action potential. Thus, on a phenomenological level, the aEIF model could possibly account for rapid spike initiation in real neurons.

\section{Acknowledgments}

This work was supported by Swiss National Science Foundation Grant number SNF 200020-108093/1.

\section{References}

[1] R. Brette, W. Gerstner, Adaptive exponential integrate-and-fire model as an effective description of neuronal activity, J. Neurophysiol. 94 (2005) 3637-3642.

[2] N. Fourcaud-Trocmé, D. Hansel, C. van Vreeswijk, N. Brunel, How spike generation mechanisms determine the neuronal response to fluctuating inputs, J. Neurosci. 23 (2003) 11628-11640.

[3] W. Gerstner, W. Kistler, Spiking neuron Models: Single Neurons, Populations, Plasticity, Cambridge University Press, Cambridge, 2002.

[4] M.S. Goldman, J. Golowasch, E. Marder, L.F. Abbott, Global structure, robustness and modulation of neuronal models, J. Neurosci. 21 (2001) 5229-5238.

[6] A. Hodgkin, A. Huxley, A quantitative description of membrane current and its application to conduction and excitation in nerve, J. Physiol. 117 (1952) 500-544.

[7] E. Izhikevich, Which model to use for cortical spiking neurons IEEE Trans. Neural Networks 15 (2004) 1063-1070.

[8] R. Jolivet, A. Rauch, H.-R. Lüscher, W. Gerstner, Predicting spike timing of neocortical pyramidal neurons by simple threshold models, J. Comput. Neurosci. 21 (2006) 35-49.

[9] W. Kistler, W. Gerstner, J. van Hemmen, Reduction of HodgkinHuxley equations to a single-variable threshold model, Neural. Comput. 9 (1997) 1015-1045.

[10] L. Ljung, System Identification: Theory for the User, second ed., Prentice-Hall, Englewood Cliffs, NJ, 1999.

[11] Z. Mainen, T. Sejnowski, Reliability of spike timing in neocortical neurons, Science 268 (1995) 1503-1506.

[12] B. Naundorf, F. Wolf, M. Volgushev, Unique features of action potential initiation in cortical neurons, Nature 440 (2006) 1060-1063.

[13] A.A. Prinz, C.P. Billimoria, E. Marder, Alternative to hand-tuning conductance-based models: construction and analysis of databases of model neurons, J. Neurophysiol. 90 (2003) 3998-4015.

[14] A. Rauch, G. La Camera, H.-R. Lüscher, W. Senn, S. Fusi, Neocortical pyramidal cells respond as integrate-and-fire neurons to in vivo-like input currents, J. Neurophysiol. 90 (2003) 1598-1612.

[15] M.J. Richardson, N. Brunnel, V. Hakim, From subthreshold to firing-rate resonance, J. Neurophysiol. 89 (2003) 2538-2554.

Fig. 5. The lower four spike trains of each graph are experimental voltage traces recorded in response to the same driving current. The top trace is the simulated spike train with this driving current. The traces shown in $A$ have a high intrinsic reliability and high prediction, i.e. the model is good. $B$ has high reliability but a bad prediction, i.e. the model is insufficient. In $C$ the reliability is low and the prediction is low, but the model is good since $\Gamma_{\text {eff }}>0.65$. See Fig. 4 for the $\Gamma$ values of the different cases $A, B, C$. 


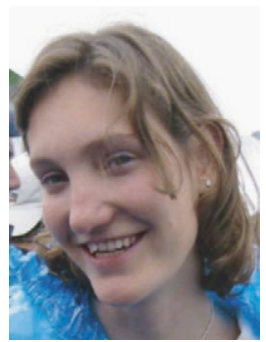

Claudia Clopath has obtained her M.Sc. in physics from the EPFL, in April 2005. She is now a Ph.D. in the Laboratory of Computational Neuroscience at the EPFL and her current research interest is to describe neuronal activity with simple Integrate-and-Fire type models.

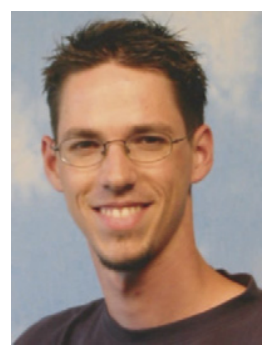

Renaud Jolivet obtained his Ph.D. in theoretical neuroscience at EPFL, in 2005, after studies in biophysics at the University of Lausanne (M.Sc.). $\mathrm{He}$ is now a postdoc in the laboratory of Prof. Pierre Magistretti at the University of Lausanne where his research is focused on neuro-astrocytic interactions in the context of brain metabolism and signal processing.

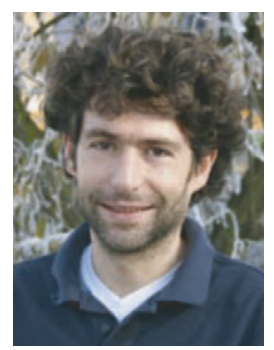

Alexander Rauch obtained his M.D., in 2003, at the University of Bern after studies in medicine at the University of Basel. His clinical experience includes surgery and neurorehabilitation. Between 1999 and 2003, he has worked as an electrophysiologist at the University of Bern focusing his research on links between cortical pyramidal neurons and simple models of neuronal activity. He is now a research assistant in the laboratory of Prof. Nikos Logothetis at the MaxPlanck-Institute where his research is focused on BOLD signal and brain imaging techniques.

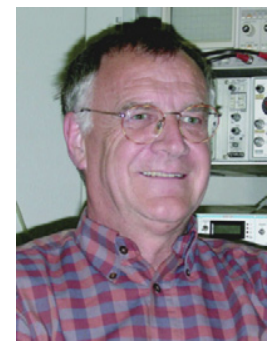

Hans-Rudolf Lüscher is Director of the Department of Physiology, University of Bern, Switzerland. He has a Medical Degree from the University of Zürich, Switzerland. He did his postdoctoral training with Elwood Henneman at the Department of Physiology and Biophysics, Harvard Medical School, Boston, USA. He spent extended time periods at the Division of Neuroscience, John Curtin School of Medical Research, Canberra, Australia. Hans-Rudolf Lüscher served as Dean and Vice-Dean of the Medical Faculty, University of Bern. He is a member of the Science Council of the Swiss National Science Foundation and of the Swiss Academy of Medical Sciences.

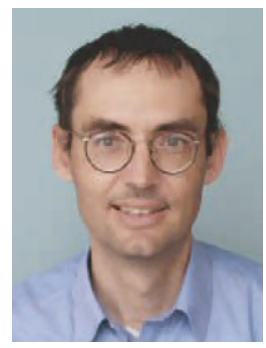

Wulfram Gerstner received his Ph.D. degree in theoretical physics from the TU Munich, Germany, in 1993, after studies in Tübingen, Berkeley, and Munich. He is a Professor and Head of Laboratory of Computational Neuroscience, EPFL, Switzerland. 